\title{
Implementación de balón de resucitación aórtico endovascular (REBOA) para el control de hemorragia no compresible de torso
}

\author{
Juan Pablo Ramos P. ${ }^{1}$, Javier Vela U. ${ }^{2}$, Analia Zinco A. ${ }^{1}$, \\ Pablo Achurra T. ${ }^{2}$, Julián Varas ${ }^{2}$ y Pablo Ottolino L. ${ }^{1}$
}

Unidad de Trauma y Urgencias Hospital Dr. Sótero del Río. Santiago, Chile. Departamento de Cirugía Pontificia Universidad Católica de Chile. Santiago, Chile

Recibido 2021-01-31 y aceptado 2021-03-10

Correspondencia a: Dr. Juan Pablo Ramos P. jramos.med@gmail.com

\section{Implementation of the resuscitative endovascular balloon occlusion of the aorta (REBOA) for the control of non-compressible torso hemorrhage}

\begin{abstract}
Introduction: Non-compressible torso hemorrhage currently has a high morbidity and mortality even in the most specialized referral centers. REBOA is an emerging tool that is used as early hemostatic control in this type of patient. Clinical Case: We present the case of a 25-year-old female patient who suffers severe pelvic trauma after falling from a height. He was admitted hemodynamically unstable, for which a massive transfusion protocol was activated and a right common femoral arterial access was performed. After presenting a transient response to resuscitation, a REBOA balloon was installed in zone 3, increasing systolic pressure up to $130 \mathrm{mmHg}$, later transferring to the operating room. Preperitoneal pelvic packing and external fixation were performed, deflating the balloon after 29 minutes in zone 3 . The patient left the intensive care unit without vasoactive drugs, to complete surgery 48 hours later and definitive fixation 6 days later. The patient evolves in good general condition.
\end{abstract}

Key words: pelvic trauma; resuscitative endovascular balloon occlusion of the aorta; REBOA.

\section{Resumen}

Introducción: La hemorragia no compresible de torso, actualmente tiene una alta morbimortalidad aún en los centros de referencia más especializados. El REBOA es una herramienta emergente que se utiliza como control hemostático precoz en este tipo de pacientes. Caso Clínico: Presentamos el caso de una paciente femenina de 25 años que sufre un trauma pélvico grave tras caer de altura. Ingresa hemodinámicamente inestable por lo cual se activa protocolo de transfusión masiva y realiza acceso arterial femoral común derecho. Al presentar una respuesta transitoria a la reanimación, se instala balón de REBOA en zona 3 , logrando aumentar presión sistólica hasta $130 \mathrm{mmHg}$, trasladando posteriormente a quirófano. Se realiza packing pélvico preperitoneal y fijación externa, desinflando el balón después de 29 min en zona 3 . La paciente sale a unidad de cuidados intensivos sin drogas vasoactivas, para completar cirugía a las $48 \mathrm{~h}$ y fijación definitiva 6 días después. La paciente evoluciona en buenas condiciones generales.

Palabras clave: trauma pélvico; balón de resucitación aórtico endovascular; REBOA.

\section{Introducción}

El balón de resucitación aórtico endovascular (REBOA, siglas en inglés) es una técnica emergente que se utiliza para el control temporal de la hemorragia no compresible de torso (HNCT) en pacientes que se encuentran en una condición extrema ${ }^{1}$. El abordaje tradicional para el control de la hemorragia en estos casos, es mediante una toracotomía de resucitación junto con el clampeo de la aorta descendente ${ }^{1}$. Dentro de las causas de HNCT se encuentra el sangrado retroperitoneal secundario a una fractura de pelvis, el que se asocia a una elevada morbimortalidad hasta en los centros más especializados ${ }^{2}$. Dentro de las alternativas para el control hemostático se describe: la fijación pélvica, el packing pélvico preperitoneal y técnicas endovasculares como angioembolización y el uso del REBOA ${ }^{3}$. 
En nuestra institución, el uso de este dispositivo se enmarca en un protocolo de atención de trauma que contempla la capacitación a cirujanos del servicio de emergencia adulto por parte de la unidad de trauma y urgencia de nuestro hospital en conjunto con el Centro de Simulación de la Universidad Católica y referentes internacionales como capacitadores. Este protocolo también describe los pacientes que se benefician de este dispositivo como son los pacientes con fractura de pelvis e inestabilidad hemodinámica. A continuación, se describe un caso clínico donde se utiliza REBOA para el control temporal de la hemorragia no compresible de torso.

\section{Caso Clínico}

Paciente femenino, de 25 años, ingresa a box de trauma, traído por personal prehospitalario (SAMU), sin antecedentes mórbidos, tras sufrir caída de altura de 10 metros aproximadamente. A la evaluación primaria, con vía aérea permeable, ventilación simétrica, con saturación de $\mathrm{O} 2$ de $95 \%$, presión arterial de 50/30 mmHg, frecuencia cardiaca de $130 \mathrm{lpm}$ y Glasgow de 15 Ptos. Al examen físico se objetiva extremidad inferior izquierda rotada y acortada. Se realiza Extended Focused Assessment with Sonography for Trauma (EFAST) con resultado negativo, sin embargo, se inicia protocolo de transfusión masiva administrando 2 unidades de glóbulos rojos y 2 unidades de plasma fresco congelado, logrando una presión arterial de 90/50 $\mathrm{mmHg}$. Por sospecha de fractura de pelvis se instala faja pélvica y un equipo de 2 cirujanos capacitados, insertan bajo ultrasoni- do un introductor 4 French $(F r)$ en arteria femoral común derecha.

Se traslada a tomografía computada con protocolo split bolus para paciente inestable en donde no se evidencian lesiones en cráneo, tórax, ni intraabdominal. En pelvis, se confirma fractura tipo VS izquierda, con hematoma presacro con extensión retroperitoneal y fractura de fémur izquierdo proximal (Figura 1A).

A pesar de una reanimación continúa con hemoderivados, solo se logra una respuesta hemodinámica transitoria por lo cual se decide la instalación de balón de REBOA (REBOA Balloon Kit ${ }^{\mathrm{TM}} 20 \times 30$. REBOA Medical. Distribuido por CMS Medical Chile), utilizando el acceso arterial previo cambiando a un introductor 7 Fr (Figura 1B). Se infla balón de REBOA en zona III logrando repunte de presión a $134 / 83 \mathrm{mmHg}$, con lo cual se traslada al paciente para exploración quirúrgica.

En quirófano, con aorta infrarrenal ocluida, se realiza abordaje pélvico extraperitoneal, evacuando abundante contenido hemático $\mathrm{y}$, posteriormente, se realiza packing preperitoneal con 5 compresas (3 izquierdas 2 derechas). Luego de esta maniobra, se procede a desinflar el balón de forma gradual, marcando un tiempo total de oclusión de $29 \mathrm{~min}$. Tras el desinflado, la paciente mantiene presiones sistólicas sobre $100 \mathrm{mmHg}$, por lo cual se procede a realizar fijación pélvica externa (Figura 2A) y fijación transarticular de cadera izquierda, manteniendo el acceso arterial y el balón desinflado (Figura 2B).

Después de 65 min se retira balón, guía e introductor y se realiza angiografía a extremidad inferior derecha objetivando adecuada permeabilidad distal (Figura 3).

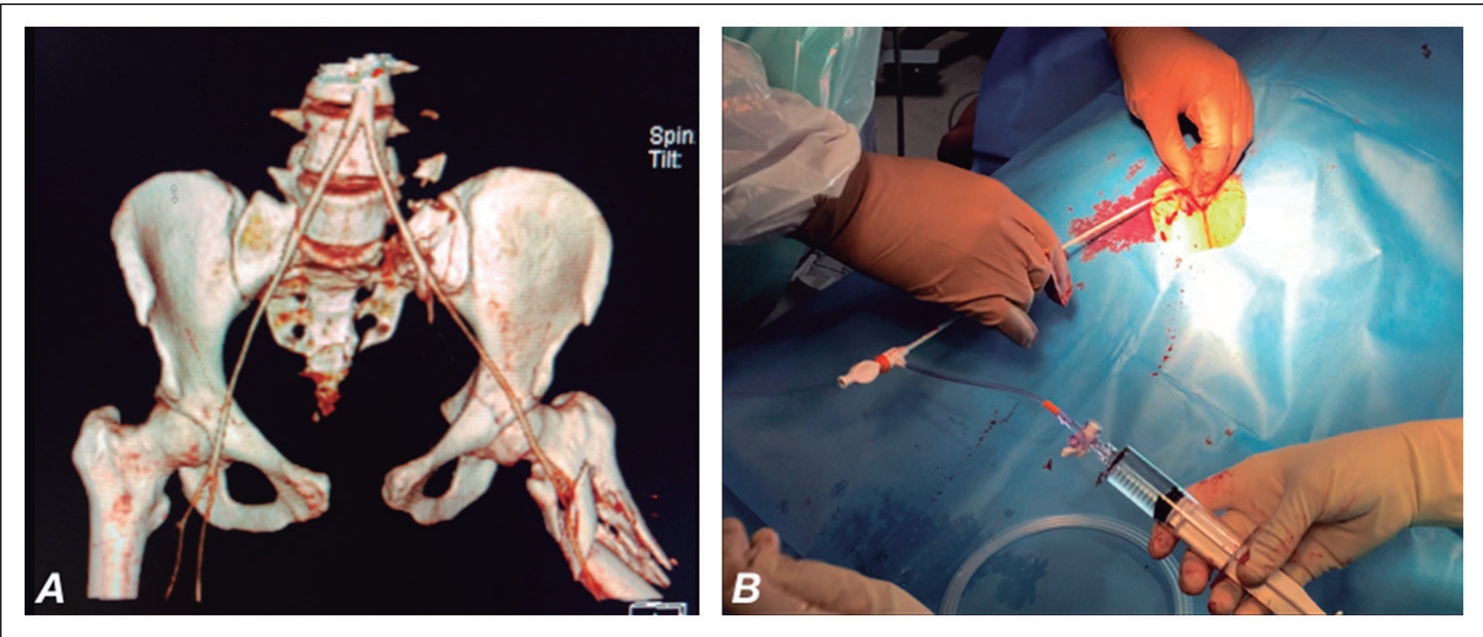

Rev. Cir. 2021;73(4):514-518
Figura 1: A: Reconstrucción 3D de fractura de pelvis tipo VS. B: Instalación de vaina 7 Fr por acceso arterial previo $4 \mathrm{Fr}$ instalado bajo ultrasonido en arteria femoral común derecha. 


\section{CASOS CLínICOS}
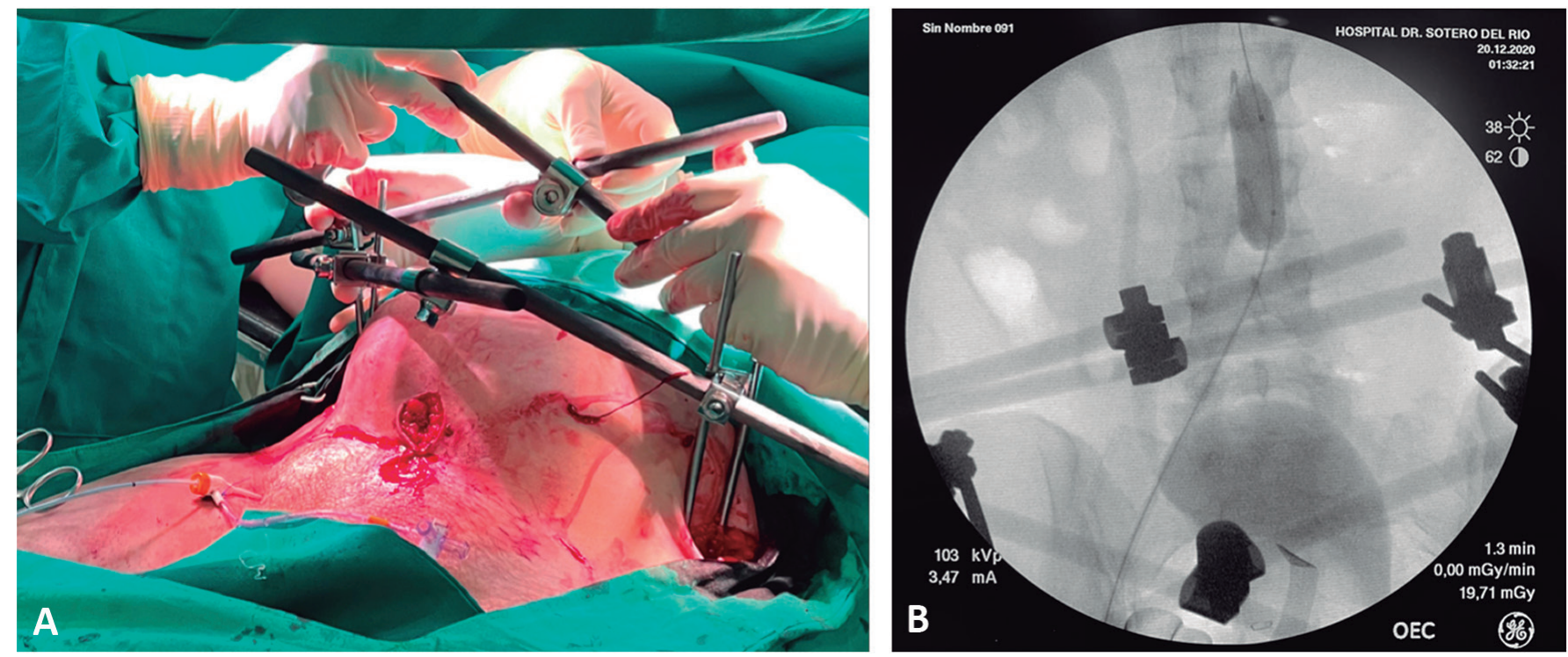

Figura 2. A: Paciente con oclusión en zona III por REBOA, además de packing preperitoneal mientras se instala fijación externa de pelvis. B: Paciente en quirófano, posterior a packing pélvico preperitoneal, fijación externa y oclusión aórtica.

Figura 3. Angiografía de la extremidad inferior derecha, previo al retiro de la vaina $7 \mathrm{Fr}$. Esta se encuentra normal hasta arco plantar.

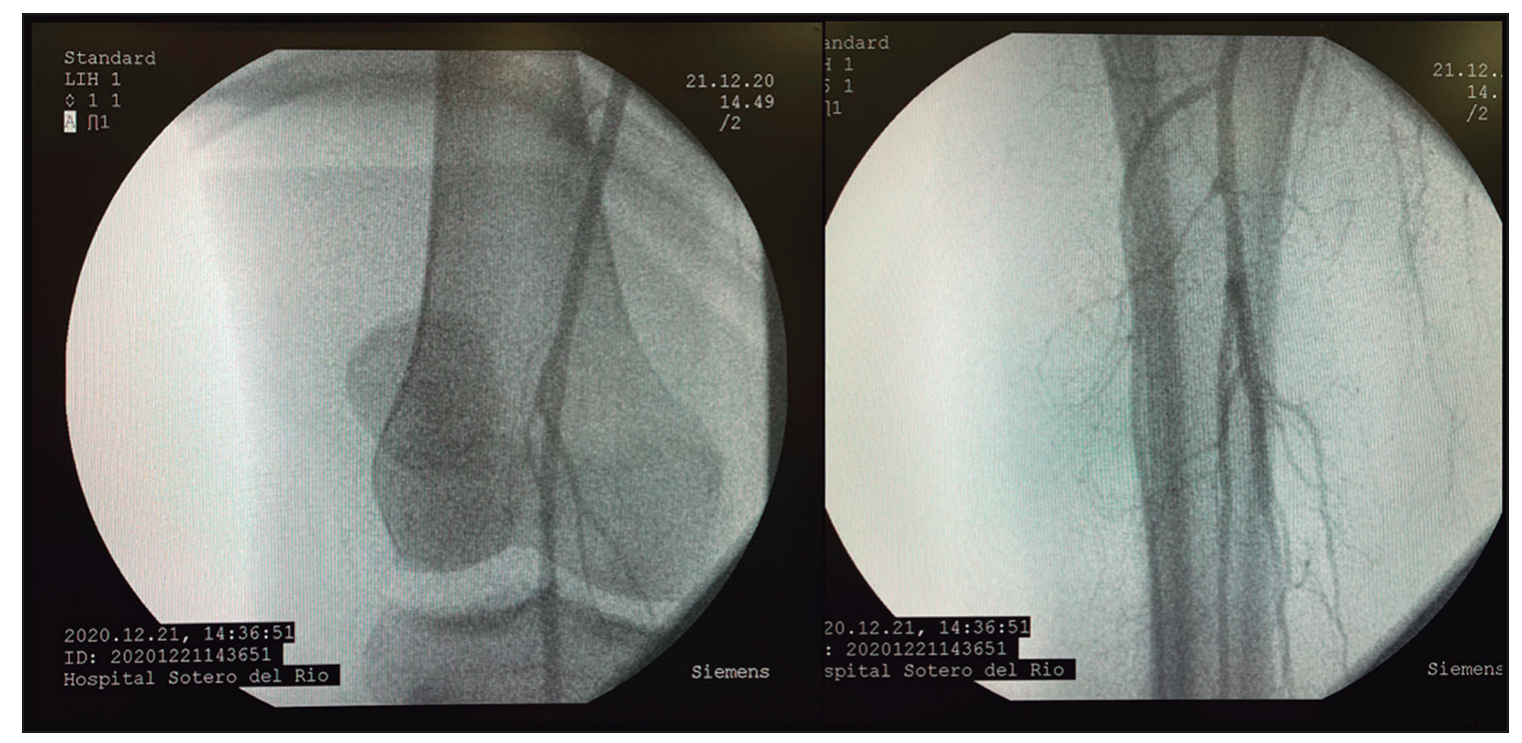

Se realiza tromboelastometría con parámetros CT $51 \mathrm{seg}$, CFT $205 \mathrm{seg}$, MCF $50 \mathrm{~mm}$, ML 3\% y decide traslado a unidad de cuidados intensivos donde evoluciona estable sin requerimientos de drogas vasoactivas. En total los requerimientos transfusionales de las primeras $24 \mathrm{~h}$ fueron de 6 UGR, 6 UPFC, 6 UPLQT, 12 U Crio. A las 24 h, se realiza cirugía de second look, retirándose packing preperitoneal sin objetivar sangrado e instalando material hemostático en preperitoneo y cierre aponeurótico. La paciente evoluciona favorablemente en unidad de cuidados intensivos, y pese a presentar tromboembolismo pulmonar, logra ser extubada al día 7 de hospitalización para, posteriormente, realizar fijación definitiva por el equipo de traumatología. Se da de alta en buenas condiciones para continuar rehabilitación motora. 


\section{Discusión}

Este es el primer reporte del uso de REBOA en Chile para control temporal de HNCT de origen traumático, utilizando un perfil bajo de 7 Fr como acceso arterial, según la revisión realizada a la fecha. Si bien esta técnica ha sido utilizada en pacientes con aneurisma de aorta abdominal roto (con otro tipo de balones e introductores), existe escasa experiencia en nuestro país en el ámbito del trauma. Los mismos autores han utilizado esta técnica con introductor 14 Fr y balón Coda (Cateter Balón Coda $^{\circledR}$ de Cook Medical) en el 2019 observando grandes diferencias entre ambas, principalmente en la facilidad y rapidez del acceso arterial con introductor $7 \mathrm{Fr}$. Actualmente REBOA es una herramienta emergente de control hemostático precoz de la HNCT del paciente que ingresa con inestabilidad hemodinámica a un centro especializado ${ }^{4-6}$.

El mecanismo fisiológico que beneficia a estos pacientes se basa en la redistribución del flujo sanguíneo generado por la oclusión aórtica, logrando aumentar la perfusión miocárdica y cerebral ${ }^{7,8}$, además de disminuir el flujo hacia el territorio con hemorragia activa por debajo del nivel de oclusión. El entrenamiento y protocolización son puntos claves para lograr este beneficio en la reanimación y disminuir el desarrollo de complicaciones. Estas últimas pueden ocurrir tanto en el acceso arterial, como en la reperfusión que se puede desencadenar como consecuencia de la isquemia en los tejidos distales a la oclusión. El nivel de oclusión del REBOA dependerá de la indicación y del origen del sangrado, existiendo 2 zonas anatómicas a utilizar, la zona I que se encuentra entre la arteria subclavia izquierda y el tronco celíaco, y la zona III, desde las arterias renales hasta la bifurcación de la aorta ${ }^{9,10}$.

Las principales limitaciones para el uso de este dispositivo incluyen su disponibilidad y la necesidad de un entrenamiento adecuado en su uso. El uso del REBOA obedece a eventos de baja incidencia, que ocurren en condiciones de extrema emergencia donde no pueden permitirse errores ${ }^{11}$. Este procedimiento, por lo tanto, no es idóneo para ser enseñado con detenimiento en un contexto clínico real, sino que resulta más adecuado el aprendizaje en un ambiente simulado. El entrenamiento mediante simulación provee la oportunidad de realizar una práctica deliberada y continua $^{12}$. Eso último resulta fundamental, pues se ha visto que, tras un curso de entrenamiento, en ausencia de casos clínicos de REBOA, las habilidades adquiridas empiezan a perderse a los 6 meses $^{13}$. El Centro de Simulación de la Pontificia Universidad Católica junto a la Unidad de Trauma y Urgencia del Complejo Asistencial Sótero del Río desarrollaron un modelo de bajo costo, al que se le puede hacer ultrasonido y punción para el entrenamiento en el uso de este dispositivo, presentado durante el Workshop de REBOA el 5-6 de marzo de 2020 en la misma institución (datos por publicar, Figura 4).

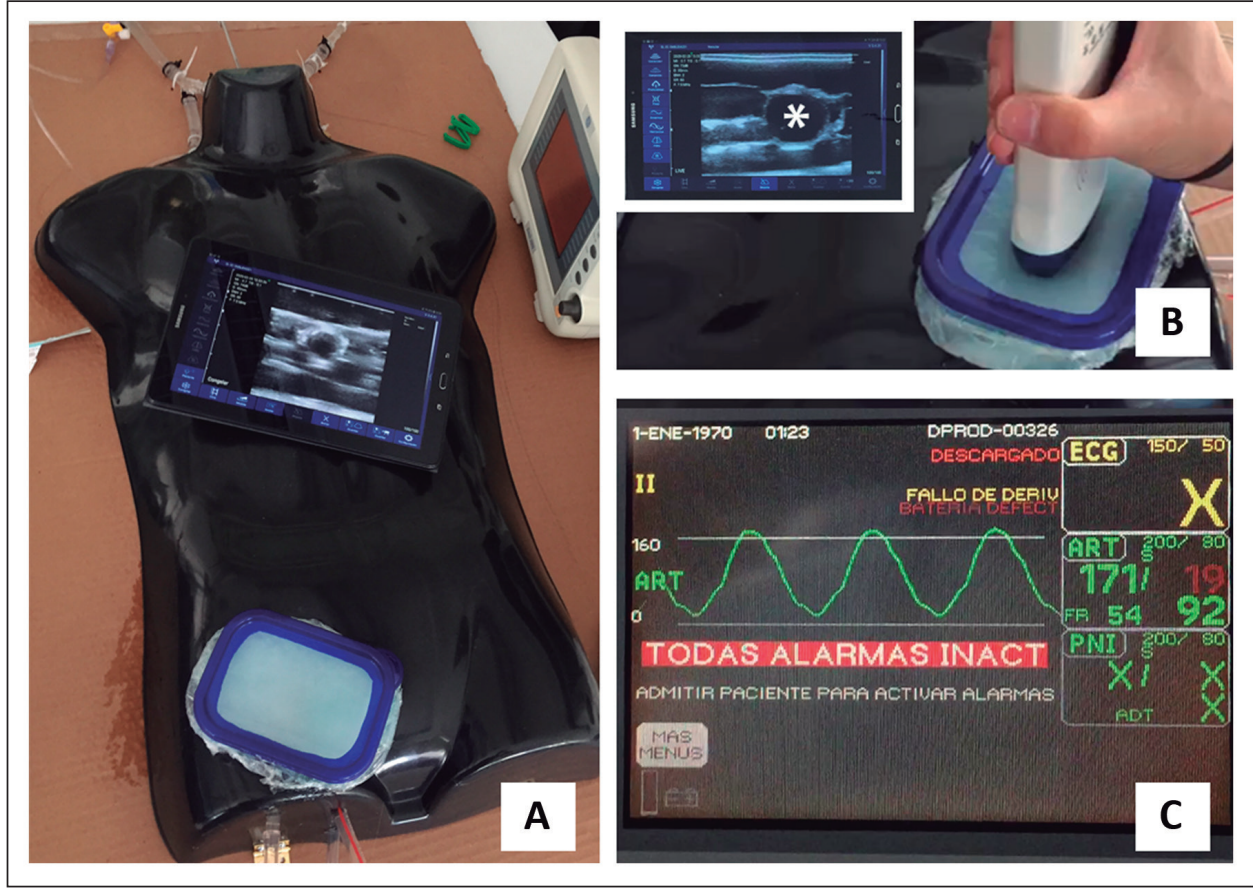

Figura 4. Modelo para entrenamiento en uso de REBOA elaborado en el Centro de Simulación de la Universidad Católica. A: Modelo: en la región inguinal derecha tiene un acceso vascular ecografiable y puncionable. B: Ecografía de la región inguinal, $\left(^{*}\right)$ representa vaso arterial a puncionar. C: Curvas de presión obtenidas con el modelo. 
Creemos que el REBOA forma solo una parte de toda la cadena de supervivencia del paciente gravemente lesionado, al cual se agrega, la activación precoz del equipo de trauma, protocolo de transfusión masiva, cirugía de control de daños, procedimientos endovasculares y manejo intensivo. Los pacientes que obtendrán un beneficio de este procedimiento deben ser estrictamente seleccionados y su manejo altamente protocolizado.

\section{Conclusiones}

El uso del REBOA como herramienta para el control de la HNCT traumática puede ser aplicado en centros especializados nacionales. Los buenos resultados de este dispositivo se acompañan de protocolos estrictos, entrenamiento del personal y la adecuada selección del paciente.

\section{Responsabilidades éticas}

Protección de personas y animales. Los autores declaran que para esta investigación no se han realizado experimentos en seres humanos ni en animales.

Confidencialidad de los datos. Los autores declaran que en este artículo no aparecen datos de pacientes. local.

Este artículo tiene aprobación del comité de ética

Conflictos de interés: no hay.

\section{Bibliografía}

1. Morrison JJ, Rasmussen TE.

Noncompressible Torso Hemorrhage. A

Review with Contemporary Definitions and Management Strategies. Surg

Clin North Am. 2012;92:843-58.

doi: $10.1016 /$ j.suc.2012.05.002.

2. Kisat M, Morrison JJ, Hashmi ZG, Efron DT, Rasmussen TE, Haider AH. Epidemiology and outcomes of noncompressible torso hemorrhage. J Surg Res. 2013;184:414-21. doi: 10.1016/j. jss.2013.05.099.

3. Stannard A, Eliason JL, Rasmussen TE. Resuscitative endovascular balloon occlusion of the aorta (REBOA) as an adjunct for hemorrhagic shock. J Trauma - Inj Infect Crit Care 2011;71:1869-72. doi: 10.1097/TA.0b013e31823fe90c.

4. Gupta BK, Khaneja SC, Flores L, Eastlick L, Longmore W, Shaftan GW. The role of intra-aortic balloon occlusion in penetrating abdominal trauma. J Trauma

- Inj Infect Crit Care 1989;29:861-5. doi: 10.1097/00005373-19890600000026 .

5. Low RB, Longmore W, Rubinstein R, Flores L, Wolvek S. Preliminary report on the use of the percluder ${ }^{\circledR}$ occluding aortic balloon in human beings. Ann Emerg Med. 1986;15:1466-9. doi: 10.1016/ S0196-0644(86)80945-3.

6. Bukur M, Warnack E, DiMaggio C, Frangos S, Morrison JJ, Scalea TM, et al. Temporal Changes in Reboa Utilization Practices are Associated with Increased Survival: An Analysis of the Aorta Registry. Shock. 9000; Publish Ah https://journals.lww.com/shockjournal/ Fulltext/9000/Temporal_Changes_in Reboa_Utilization_Practices.97398.aspx.

7. Markov NP, Percival TJ, Morrison JJ, Ross JD, Scott DJ, Spencer JR, et al. Physiologic tolerance of descending thoracic aortic balloon occlusion in a swine model of hemorrhagic shock. Surg (United States). 2013;153:848-56. doi: 10.1016/j.surg.2012.12.001.

8. Teixeira PGR, Inaba K, Hadjizacharia P, Brown C, Salim A, Rhee P, et al. Preventable or potentially preventable mortality at a mature trauma center. J Trauma. 2007;63:1338-47. doi: 10.1097/ TA.0b013e31815078ae.

9. Biffl WL, Fox CJ, Moore EE. The role of REBOA in the control of exsanguinating torso hemorrhage. J Trauma Acute Care Surg. 2015;78:1054-8. doi: 10.1097/ TA.0000000000000609.
10. Vrancken SM, Borger van der Burg BLS, Vrancken PJEM, Kock GAH, Rasmussen TE, Hoencamp R. A contemporary assessment of devices for Resuscitative Endovascular Balloon Occlusion of the Aorta (REBOA): resource-specific options per level of care. Eur J Trauma Emerg Surg. Published online 2020. doi: 10.1007/ s00068-020-01382-5.

11. Engberg M, Taudorf M, Rasmussen NK, Russell L, Lönn L, Konge L.

Training and assessment of competence in resuscitative endovascular balloon occlusion of the aorta (REBOA) - a systematic review. Injury 2020;51:147-56. doi: 10.1016/j. injury.2019.11.036.

12. Anders Ericsson K. Deliberate Practice and Acquisition of Expert Performance: A General Overview. Acad Emerg Med. 2008;1:988-994. doi: 10.1111/j.15532712.2008.00227.x.

13. Hatchimonji JS, Sikoutris J, Smith BP, Vella MA, Dumas RP, Qasim ZA, et al. The REBOA Dissipation Curve: Training Starts to Wane at 6 Months in the Absence of Clinical REBOA Cases. J Surg Educ. Published online July 30, 2020. doi: 10.1016/j. jsurg.2020.05.003 